Supporting Information for:

\title{
Amphiphilicity in Oxygen Atom Transfer Reactions of
}

\section{Oxobis(iminoxolene)osmium Complexes}

Alexander N. Erickson, Jacqueline Gianino, Sean J. Markovitz, and Seth N. Brown*

\author{
251 Nieuwland Science Hall \\ Department of Chemistry and Biochemistry \\ University of Notre Dame \\ Notre Dame, Indiana 46556-5670 USA \\ E-mail: Seth.N.Brown.114@nd.edu
}

Contents:

I. $\quad{ }^{31}$ P NMR spectra

Figure S1. ${ }^{31} \mathrm{P}$ NMR spectra of $\left({ }^{\mathrm{H}} \mathrm{ap}\right)_{2} \mathrm{Os}\left(\mathrm{P}[\mathrm{OAr}]_{3}\right)_{2}$

II. X-ray crystallography

A. Figure S2. Thermal ellipsoid plot of $c i s-\beta-\left({ }^{\mathrm{H}} \text { ap }\right)_{2} \mathrm{Os}\left(\mathrm{P}[\mathrm{O}-\mathrm{o}-\mathrm{Tol}]_{3}\right)\left(\mathrm{NCCH}_{3}\right) \quad \mathrm{S} 3$

B. Figure S3. Thermal ellipsoid plot of trans- $\left({ }^{\mathrm{H}} \text { ap }\right)_{2} \mathrm{Os}(\mathrm{CNXy})_{2} \quad \mathrm{~S} 4$

III. Kinetics

A. Reactions of $\left({ }^{\mathrm{H}} \mathrm{ap}\right)_{2} \mathrm{OsO}$ with $\mathrm{PAr}_{3}$

Figure S4. $k_{\text {obs }}$ vs. [PAr 3 ]

S5

Table S1. Second-order $k_{\mathrm{OAT}}$ for reaction of $\mathrm{PAr}_{3}$ with $\left({ }^{\mathrm{H}} \mathrm{ap}\right)_{2} \mathrm{OsO} \quad$ S5

B. Reactions of $\left({ }^{\mathrm{R}} \text { ap }\right)_{2} \mathrm{OsO}$ with $\mathrm{PPh}_{3}$

Figure S5. $k_{\text {obs }}$ Vs. $\left[\mathrm{PPh}_{3}\right] \quad$ S6

Table S2. Second-order $k_{\mathrm{OAT}}$ for reaction of $\mathrm{PPh}_{3}$ with $\left({ }^{\mathrm{R}} \text { ap }\right)_{2} \mathrm{OsO} \quad \mathrm{S} 6$

C. Reactions of $\left({ }^{\mathrm{R}} \text { ap }\right)_{2} \mathrm{OsO}$ with $\mathrm{P}(\mathrm{O}-o-\mathrm{Tol})_{3}$

Figure S6. $k_{\text {obs }}$ vs. [P(O-o-Tol $\left.)_{3}\right] \quad S 7$

Table S3. Second-order $k_{\mathrm{OAT}}$ for reaction of $\mathrm{P}(\mathrm{O}-o-\mathrm{Tol})_{3}$ with $\left({ }^{\mathrm{R}} \mathrm{ap}\right)_{2} \mathrm{OsO} \quad \mathrm{S} 7$

IV. Energies, MOS Values, and Cartesian Coordinates from DFT Calculations
A. (ap) $)_{2} \mathrm{OsO}$
S8
B. trans-(ap) $)_{2} \mathrm{Os}\left(\mathrm{PMe}_{3}\right)_{2}$
S9
C. $(\text { ap })_{2} \mathrm{Os}\left(\mathrm{PMe}_{3}\right)$
S11
D. $c i s-\beta-(\operatorname{ap})_{2} \mathrm{Os}\left(\mathrm{P}[\mathrm{OMe}]_{3}\right)_{2}$
$\mathrm{S} 12$
E. Transition state for addition of $\mathrm{PMe}_{3}$ to (ap) $)_{2} \mathrm{OsO}$
S14
F. (ap) $)_{2} \mathrm{Os}\left(\mathrm{OPMe}_{3}\right)$ 


\section{I. $\quad{ }^{31}$ P NMR spectra}

Figure S1. ${ }^{31} \mathrm{P}\left\{{ }^{1} \mathrm{H}\right\}$ NMR spectra of $\left({ }^{\mathrm{H}} \mathrm{ap}\right)_{2} \mathrm{Os}\left(\mathrm{P}[\mathrm{OAr}]_{3}\right)_{2}(161.98 \mathrm{MHz})$. (a) Isolated $\left({ }^{\mathrm{H}} \text { ap }\right)_{2} \mathrm{Os}\left(\mathrm{P}[\mathrm{OPh}]_{3}\right)_{2}$, toluene-d $d_{8}$. (b) In situ generated $\left({ }^{\mathrm{H}} \mathrm{ap}\right)_{2} \mathrm{Os}\left(\mathrm{P}[\mathrm{O}-o-\mathrm{Tol}]_{3}\right)_{2}, \mathrm{CD}_{2} \mathrm{Cl}_{2}$.

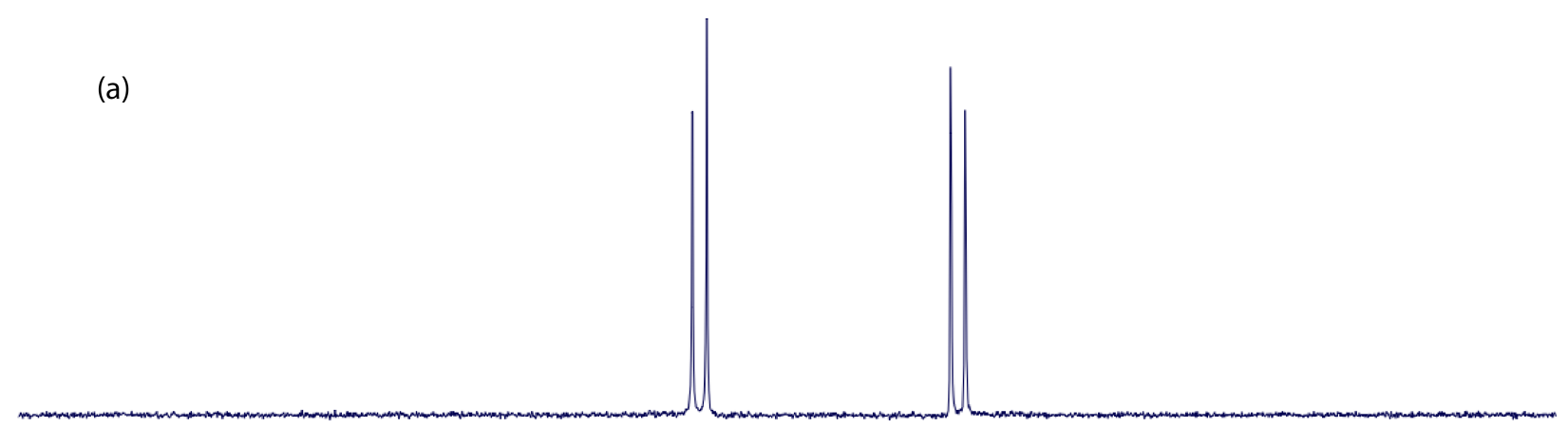

(b)

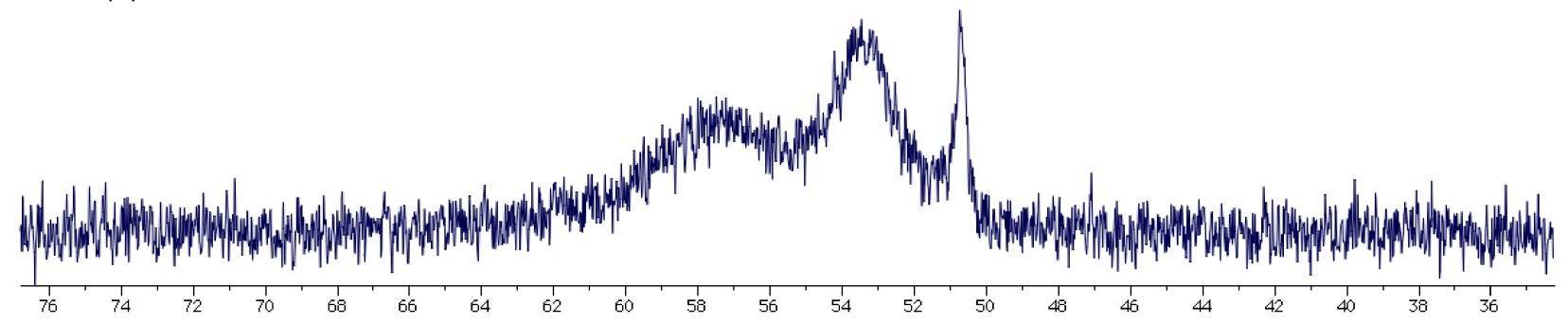




\section{X-ray crystallography}

A. Figure S2. Thermal ellipsoid plot of $c i s-\beta-\left({ }^{\mathrm{H}} \text { ap }\right)_{2} \mathrm{Os}\left(\mathrm{P}[\mathrm{O}-o-\mathrm{Tol}]_{3}\right)\left(\mathrm{NCCH}_{3}\right) \cdot \mathrm{CD}_{2} \mathrm{Cl}_{2}$ (50\% thermal ellipsoids). Hydrogen atoms and lattice solvent have been omitted for clarity. Only one orientation of each disordered tert-butyl and $o$-tolyl group is shown. (a) Molecule 1. (b) Molecule 2.

(a)

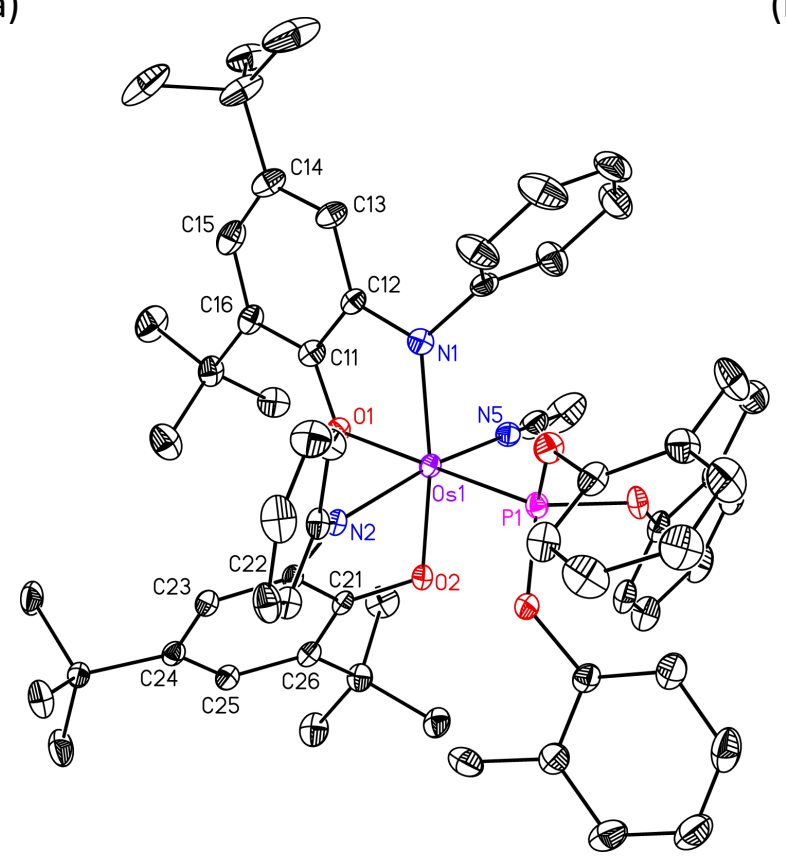

(b)

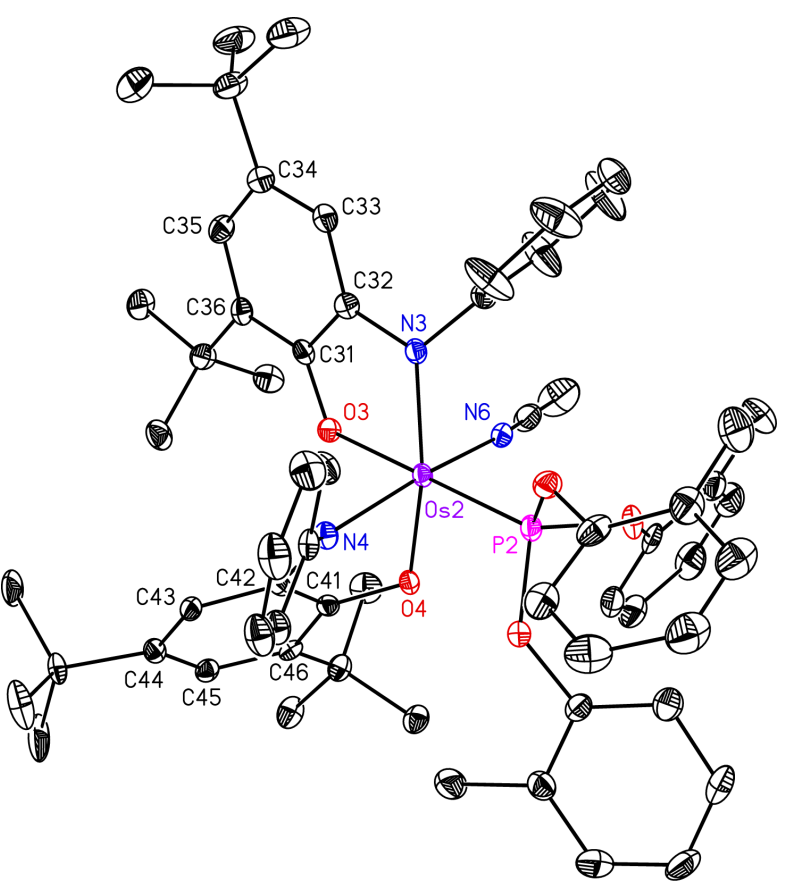


B. Figure S3. Thermal ellipsoid plot of trans $-\left({ }^{\mathrm{H}} \text { ap }\right)_{2} \mathrm{Os}\left(\mathrm{CNC}_{6} \mathrm{H}_{3}-2,6-\mathrm{Me}_{2}\right)_{2} \cdot 2 \mathrm{CD}_{2} \mathrm{Cl}_{2}$. Hydrogen atoms and lattice solvent have been omitted for clarity.

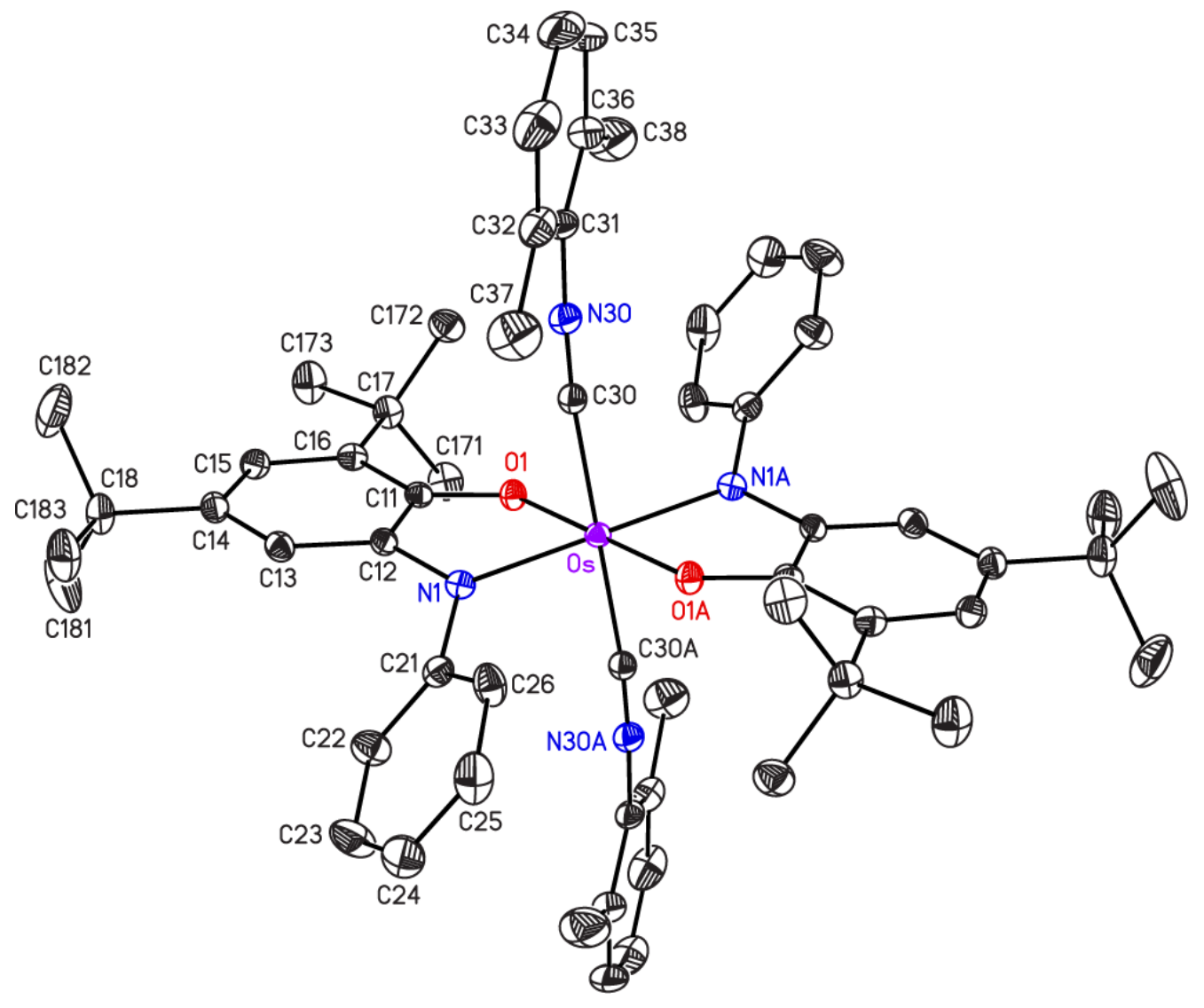




\section{Kinetics}

\section{A. Reactions of $\left({ }^{\mathrm{H}} \text { ap }\right)_{2} \mathrm{OsO}$ with $\mathrm{P}\left(\mathrm{C}_{6} \mathrm{H}_{4}-p-\mathrm{X}\right)_{3}$}

Figure S4. $k_{\text {obs }}$ vs. $\left[\mathrm{P}\left(\mathrm{C}_{6} \mathrm{H}_{4}-p-\mathrm{X}\right)_{3}\right]$ for reaction with $\left({ }^{\mathrm{H}} \text { ap }\right)_{2} \mathrm{OsO}\left(\mathrm{CH}_{2} \mathrm{Cl}_{2}, 23{ }^{\circ} \mathrm{C}\right)$.

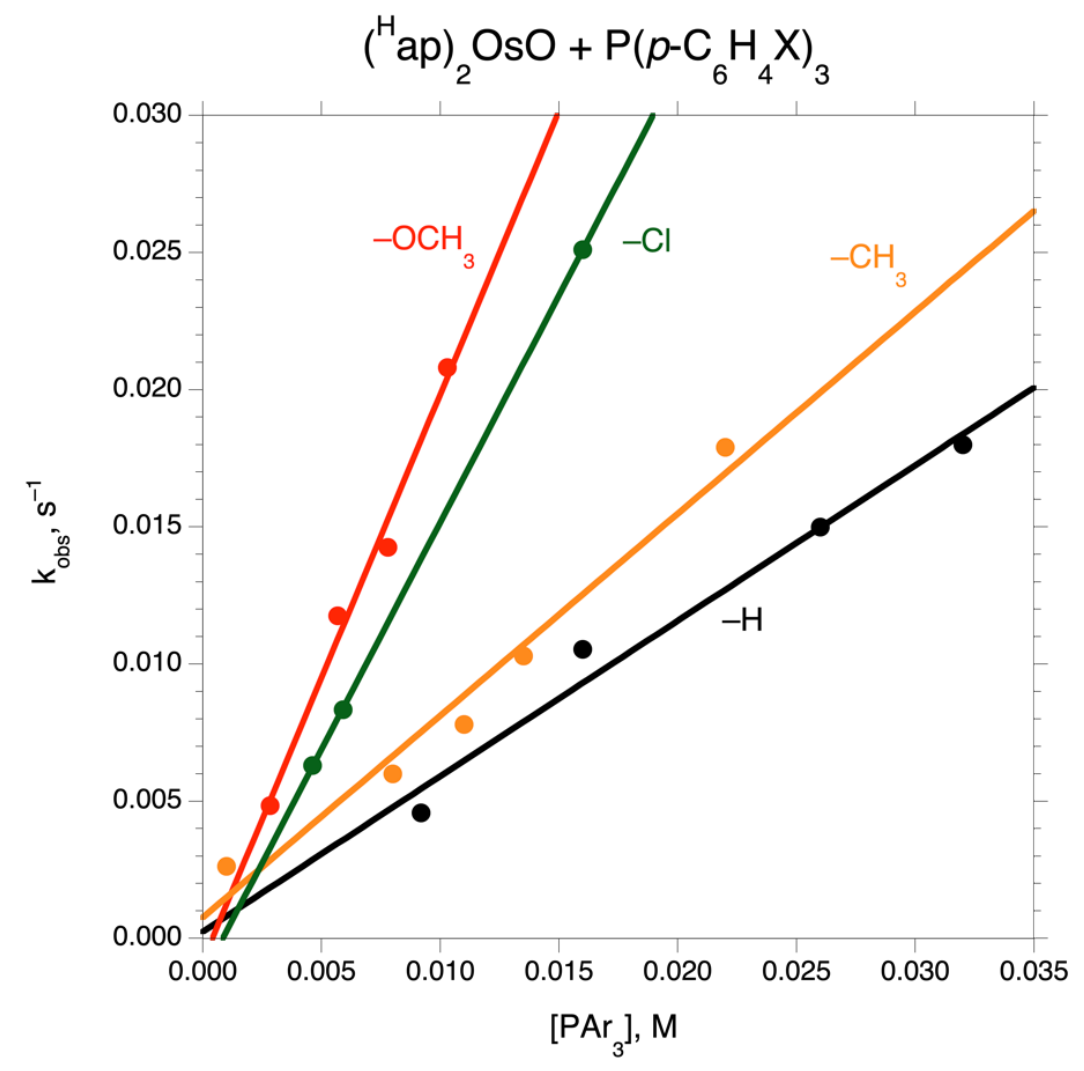

Table S1. Second-order rate constants $k_{\mathrm{OAT}}$ for reactions of $\mathrm{P}\left(\mathrm{C}_{6} \mathrm{H}_{4}-p-\mathrm{X}\right)_{3}$ with $\left({ }^{\mathrm{H}} \mathrm{ap}\right)_{2} \mathrm{OsO}$ $\left(\mathrm{CH}_{2} \mathrm{Cl}_{2}, 23{ }^{\circ} \mathrm{C}\right)$.

\begin{tabular}{|c|c|}
\hline para substituent $\mathrm{X}$ & $k_{\mathrm{OAT}}, \mathrm{L} \mathrm{mol}^{-1} \mathrm{~s}^{-1}$ \\
\hline$-\mathrm{H}$ & $0.57(6)$ \\
\hline$-\mathrm{OCH}_{3}$ & $2.07(18)$ \\
\hline$-\mathrm{CH}_{3}$ & $0.74(7)$ \\
\hline$-\mathrm{Cl}$ & $1.656(9)$ \\
\hline$-\mathrm{CF}_{3}$ & $1.003(2)$ \\
\hline \multicolumn{2}{|c|}{ Hammett $\rho=-0.02 \pm 0.14$} \\
\hline
\end{tabular}




\section{B. Reactions of $\left({ }^{\mathrm{R}} \mathrm{ap}\right)_{2} \mathrm{OsO}$ with $\mathrm{PPh}_{3}$}

Figure S5. $k_{\text {obs }}$ vs. $\left[\mathrm{PPh}_{3}\right]$ for reaction with $\left({ }^{\mathrm{R}} \mathrm{ap}\right)_{2} \mathrm{OsO}\left(\mathrm{CH}_{2} \mathrm{Cl}_{2}, 23{ }^{\circ} \mathrm{C}\right)$.

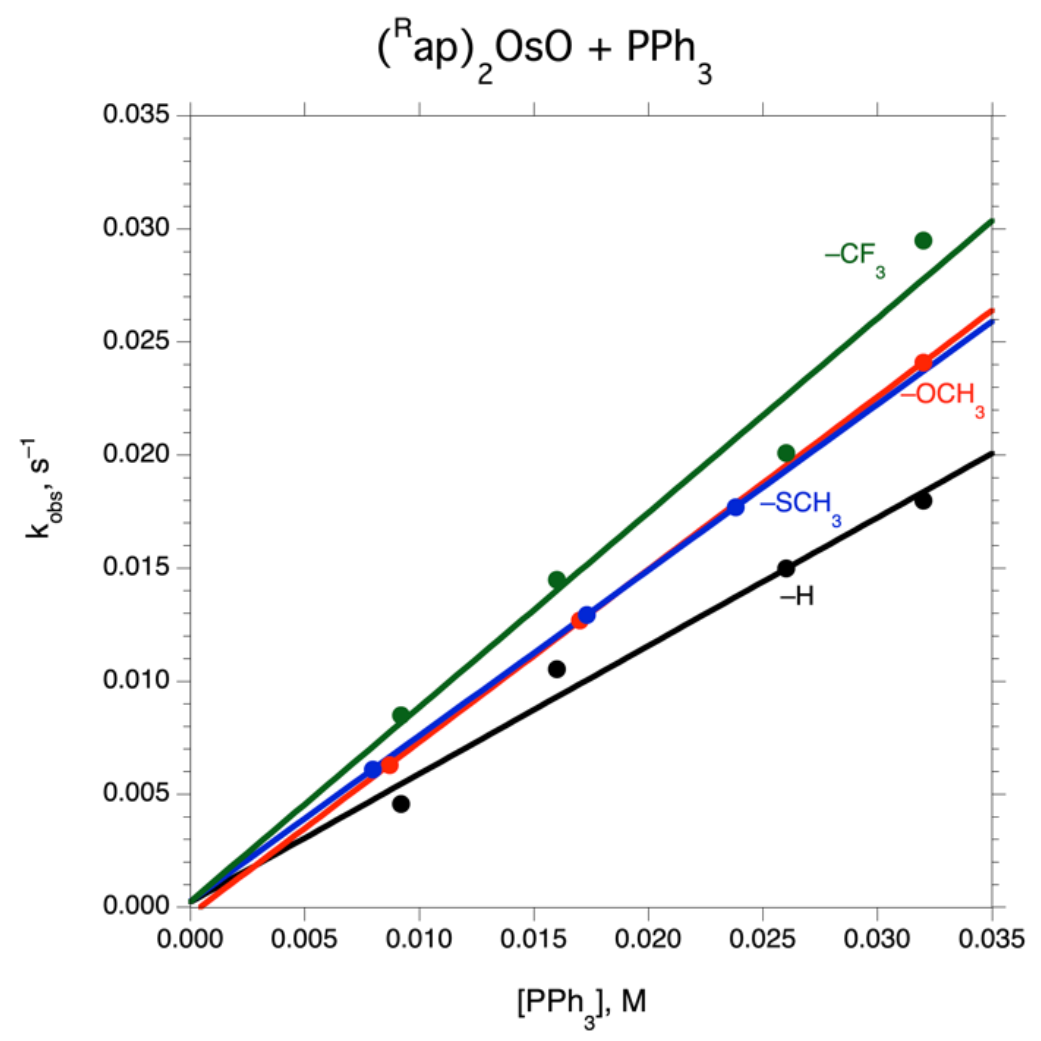

Table S2. Second-order rate constants $k_{\mathrm{OAT}}$ for reactions of $\mathrm{PPh}_{3}$ with $\left({ }^{\mathrm{R}} \text { ap }\right)_{2} \mathrm{OsO}\left(\mathrm{CH}_{2} \mathrm{Cl}_{2}\right.$, $\left.23{ }^{\circ} \mathrm{C}\right)$.

\begin{tabular}{|c|c|}
\hline para substituent $\mathrm{R}$ & $k_{\mathrm{OAT}}, \mathrm{L} \mathrm{mol}^{-1} \mathrm{~s}^{-1}$ \\
\hline$-\mathrm{H}$ & $0.57(6)$ \\
\hline$-\mathrm{SCH}_{3}$ & $0.7329(9)$ \\
\hline$-\mathrm{OCH}_{3}$ & $0.763(3)$ \\
\hline$-\mathrm{CF}_{3}$ & $0.86(12)$ \\
\hline \multicolumn{2}{|c|}{ Hammett $\rho=0.05 \pm 0.07$} \\
\hline
\end{tabular}




\section{Reactions of $\left({ }^{\mathrm{R}} \text { ap }\right)_{2} \mathrm{OsO}$ with $\mathrm{P}(\mathrm{O}-\mathrm{o}-\mathrm{Tol})_{3}$}

Figure S6. $k_{\text {obs }}$ vs. $\left[\mathrm{P}(\mathrm{O}-o-\mathrm{Tol})_{3}\right]$ for reaction with $\left({ }^{\mathrm{R}} \mathrm{ap}\right)_{2} \mathrm{OsO}\left(\mathrm{CH}_{2} \mathrm{Cl}_{2}, 23{ }^{\circ} \mathrm{C}\right)$.

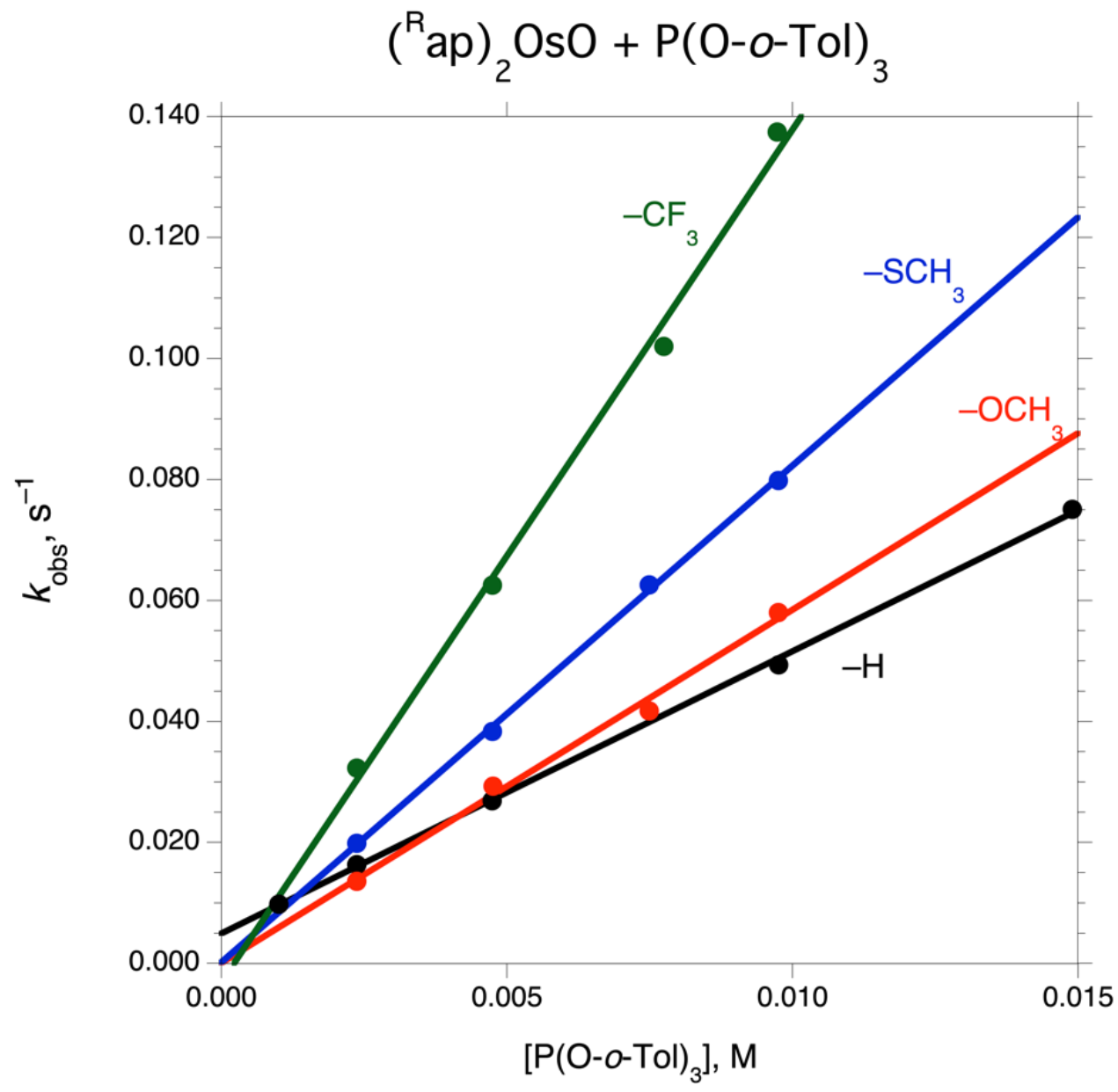

Table S3. Second-order rate constants $k_{\mathrm{OAT}}$ for reactions of $\mathrm{P}(\mathrm{O}-o-\mathrm{Tol})_{3}$ with $\left({ }^{\mathrm{R}} \text { ap }\right)_{2} \mathrm{OsO}$ $\left(\mathrm{CH}_{2} \mathrm{Cl}_{2}, 23{ }^{\circ} \mathrm{C}\right)$.

\begin{tabular}{|c|c|}
\hline para substituent $\mathrm{R}$ & $k_{\mathrm{OAT}}, \mathrm{L} \mathrm{mol}^{-1} \mathrm{~s}^{-1}$ \\
\hline$-\mathrm{H}$ & $4.66(6)$ \\
\hline$-\mathrm{SCH}_{3}$ & $8.2(2)$ \\
\hline$-\mathrm{OCH}_{3}$ & $5.8(3)$ \\
\hline$-\mathrm{CF}_{3}$ & $14.1(7)$ \\
\hline \multicolumn{2}{|c|}{ Hammett $\rho=0.26 \pm 0.12$} \\
\hline
\end{tabular}




\section{Energies, Metrical Oxidation States (MOS), and Cartesian Coordinates from DFT Calculations}

A. (ap) $)_{2} \mathrm{OsO}$

Energy of optimized structure $=-889.249747823$ a.u.

MOS $=-1.92(6)$

Cartesian coordinates of optimized structure:

\begin{tabular}{|c|c|c|c|c|}
\hline Center & Atomic & \multicolumn{3}{|c|}{ Coordinates (Angstroms) } \\
\hline Number & Number & $\mathrm{x}$ & $\mathrm{Y}$ & $\mathrm{Z}$ \\
\hline 1 & 76 & -0.000000 & -0.000027 & 0.665769 \\
\hline 2 & 8 & -0.000003 & -0.000090 & 2.378660 \\
\hline 3 & 8 & 1.407730 & -1.255878 & 0.089955 \\
\hline 4 & 7 & 1.280687 & 1.249193 & -0.085660 \\
\hline 5 & 1 & 1.034250 & 2.194586 & -0.367326 \\
\hline 6 & 6 & 2.561402 & -0.671931 & -0.344321 \\
\hline 7 & 6 & 2.515442 & 0.727435 & -0.478385 \\
\hline 8 & 6 & 3.620955 & 1.423809 & -0.971431 \\
\hline 9 & 1 & 3.585429 & 2.504616 & -1.081034 \\
\hline 10 & 6 & 4.769673 & 0.703886 & -1.309428 \\
\hline 11 & 1 & 5.638601 & 1.234412 & -1.687527 \\
\hline 12 & 6 & 4.811068 & -0.688321 & -1.167413 \\
\hline 13 & 1 & 5.712643 & -1.231699 & -1.434409 \\
\hline 14 & 6 & 3.700695 & -1.392178 & -0.691810 \\
\hline 15 & 1 & 3.709571 & -2.472416 & -0.586483 \\
\hline 16 & 8 & -1.407727 & 1.255868 & 0.090040 \\
\hline 17 & 7 & -1.280692 & -1.249188 & -0.085756 \\
\hline 18 & 1 & -1.034259 & -2.194562 & -0.367490 \\
\hline 19 & 6 & -2.561399 & 0.671958 & -0.344283 \\
\hline 20 & 6 & -2.515444 & -0.727397 & -0.478447 \\
\hline 21 & 6 & -3.620959 & -1.423732 & -0.971547 \\
\hline 22 & 1 & -3.585436 & -2.504531 & -1.081228 \\
\hline 23 & 6 & -4.769672 & -0.703780 & -1.309497 \\
\hline 24 & 1 & -5.638601 & -1.234276 & -1.687637 \\
\hline 25 & 6 & -4.811063 & 0.688416 & -1.167381 \\
\hline 26 & 1 & -5.712634 & 1.231817 & -1.434342 \\
\hline 27 & 6 & -3.700688 & 1.392235 & -0.691724 \\
\hline 28 & 1 & -3.709560 & 2.472465 & -0.586319 \\
\hline
\end{tabular}




\section{B. $\quad$ trans-(ap $)_{2} \mathrm{Os}\left(\mathrm{PMe}_{3}\right)_{2}$}

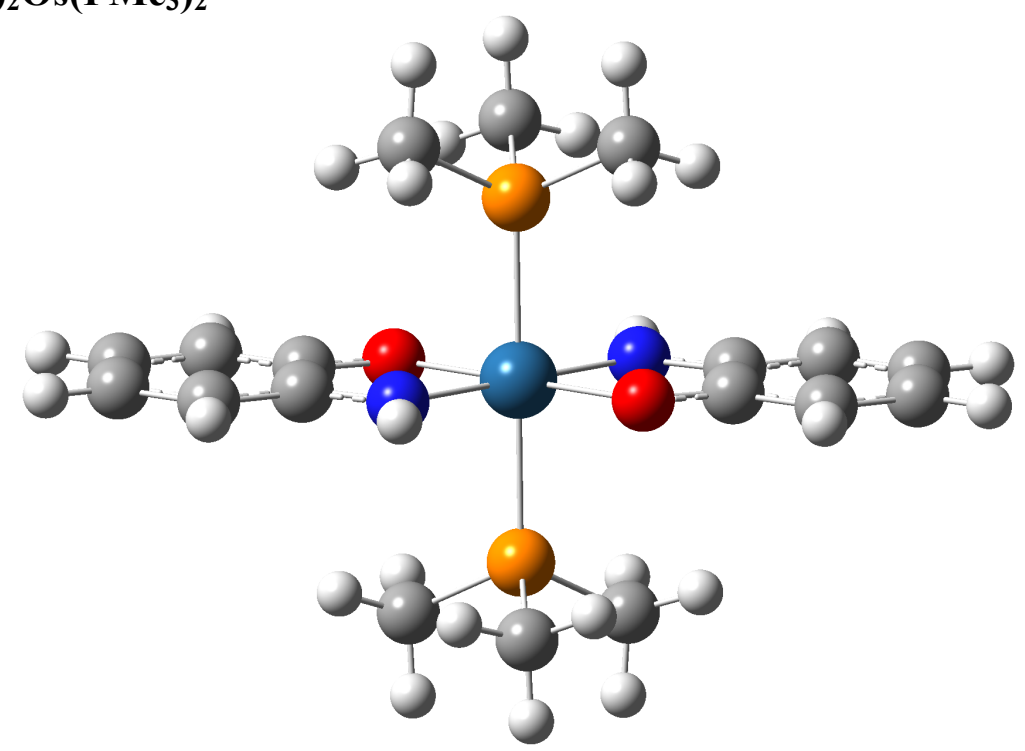

Energy of optimized structure $=-2187.64530890$ a.u.

$\operatorname{MOS}=-1.37(7)$

Cartesian coordinates of optimized structure:

\begin{tabular}{|c|c|c|c|c|}
\hline \multirow{2}{*}{$\begin{array}{l}\text { Center } \\
\text { Number }\end{array}$} & \multirow{2}{*}{$\begin{array}{l}\text { Atomic } \\
\text { Number }\end{array}$} & \multicolumn{3}{|c|}{ Coordinates (Angstroms) } \\
\hline & & $\mathrm{x}$ & $\mathrm{Y}$ & $\mathrm{Z}$ \\
\hline & & & & ---------- \\
\hline 1 & 76 & 0.084928 & 0.001348 & -0.082111 \\
\hline 2 & 8 & 1.826844 & -0.954928 & 0.662783 \\
\hline 3 & 7 & 0.272023 & -1.528444 & -1.340084 \\
\hline 4 & 1 & -0.444789 & -1.955836 & -1.921187 \\
\hline 5 & 8 & 0.408519 & 1.401071 & 1.447049 \\
\hline 6 & 7 & 1.416344 & 1.264804 & -0.949829 \\
\hline 7 & 1 & 1.464524 & 1.527325 & -1.930395 \\
\hline 8 & 15 & -1.592039 & 0.946622 & -1.297305 \\
\hline 9 & 15 & -1.436234 & -1.123117 & 1.315716 \\
\hline 10 & 6 & 2.327697 & -1.774912 & -0.235075 \\
\hline 11 & 6 & 1.498466 & -2.129016 & -1.357668 \\
\hline 12 & 6 & 1.942416 & -3.089665 & -2.293528 \\
\hline 13 & 1 & 1.300295 & -3.353372 & -3.131537 \\
\hline 14 & 6 & 3.193151 & -3.666092 & -2.145105 \\
\hline 15 & 1 & 3.548117 & -4.386698 & -2.876434 \\
\hline 16 & 6 & 4.012127 & -3.316375 & -1.049188 \\
\hline 17 & 1 & 4.993421 & -3.773223 & -0.949552 \\
\hline 18 & 6 & 3.584467 & -2.403345 & -0.095952 \\
\hline 19 & 1 & 4.202452 & -2.141387 & 0.757864 \\
\hline 20 & 6 & 1.571170 & 2.015899 & 1.271841 \\
\hline 21 & 6 & 2.112054 & 2.030189 & -0.060491 \\
\hline 22 & 6 & 3.227785 & 2.847866 & -0.346620 \\
\hline 23 & 1 & 3.619460 & 2.876349 & -1.361078 \\
\hline 24 & 6 & 3.838877 & 3.563461 & 0.671850 \\
\hline 25 & 1 & 4.718314 & 4.163945 & 0.455470 \\
\hline 26 & 6 & 3.336831 & 3.507737 & 1.988729 \\
\hline 27 & 1 & 3.831329 & 4.070668 & 2.775978 \\
\hline 28 & 6 & 2.210769 & 2.751759 & 2.287403 \\
\hline 29 & 1 & 1.796299 & 2.722908 & 3.291241 \\
\hline
\end{tabular}




$\begin{array}{lrrrr}30 & 8 & -0.981443 & 1.429216 & -2.736843 \\ 31 & 8 & -2.462462 & 2.241993 & -0.786786 \\ 32 & 8 & -2.829148 & 0.023189 & -1.825783 \\ 33 & 6 & -1.793370 & 3.338753 & -0.134267 \\ 34 & 1 & -2.577115 & 3.950838 & 0.317326 \\ 35 & 1 & -1.106654 & 2.975218 & 0.633940 \\ 36 & 1 & -1.243277 & 3.937522 & -0.868718 \\ 37 & 6 & -1.829108 & 1.966636 & -3.764472 \\ 38 & 1 & -1.165460 & 2.315928 & -4.557955 \\ 39 & 1 & -2.496817 & 1.192194 & -4.151851 \\ 40 & 1 & -2.421728 & 2.804538 & -3.383495 \\ 41 & 6 & -4.128574 & -0.013484 & -1.213731 \\ 42 & 1 & -4.669135 & -0.823020 & -1.708617 \\ 43 & 1 & -4.057508 & -0.217258 & -0.143376 \\ 44 & 1 & -4.649212 & 0.934585 & -1.374279 \\ 45 & 8 & -2.692885 & -0.264405 & 1.913783 \\ 46 & 8 & -2.259870 & -2.326943 & 0.589462 \\ 47 & 8 & -0.938202 & -1.762002 & 2.735598 \\ 48 & 6 & -3.242182 & -3.102342 & 1.299838 \\ 49 & 1 & -3.493574 & -3.944396 & 0.652241 \\ 50 & 1 & -2.837421 & -3.469585 & 2.247136 \\ 51 & 1 & -4.134333 & -2.500522 & 1.494514 \\ 52 & 6 & -2.467029 & 0.747547 & 2.916362 \\ 53 & 1 & -3.379346 & 1.346540 & 2.955014 \\ 54 & 1 & -2.292684 & 0.272812 & 3.886107 \\ 55 & 1 & -1.611770 & 1.375432 & 2.653294 \\ 56 & 6 & 0.271387 & -2.540121 & 2.851262 \\ 57 & 1 & 0.400190 & -2.733724 & 3.918235 \\ 58 & 1 & 0.161487 & -3.493045 & 2.320921 \\ 59 & 1 & 1.123471 & -1.988800 & 2.451616 \\ --------------------------------------\end{array}$




\section{C. (ap) $)_{2} \mathrm{Os}\left(\mathrm{PMe}_{3}\right)$}

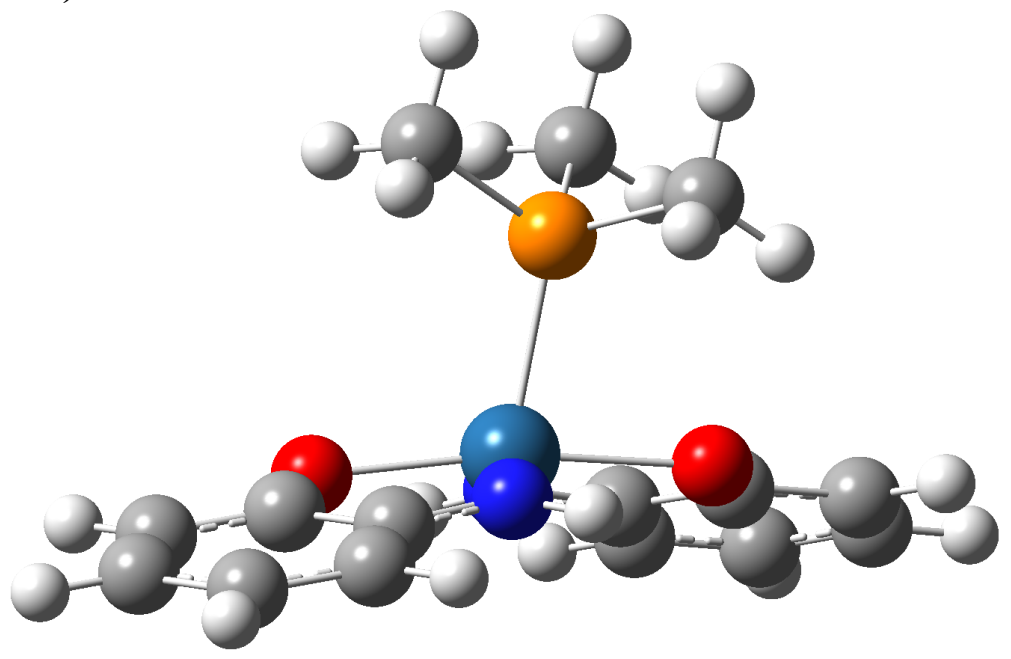

Energy of optimized structure $=-1275.15335300$ a.u.

MOS $=-1.39(5),-1.45(6)$

Cartesian coordinates of optimized structure:

\begin{tabular}{|c|c|c|c|c|}
\hline \multirow{2}{*}{$\begin{array}{l}\text { Center } \\
\text { Number }\end{array}$} & \multirow{2}{*}{$\begin{array}{l}\text { Atomic } \\
\text { Number }\end{array}$} & \multicolumn{3}{|c|}{ Coordinates (Angstroms) } \\
\hline & & $\mathrm{x}$ & $\mathrm{Y}$ & $\mathrm{Z}$ \\
\hline 1 & 76 & -0.037495 & -0.183247 & -0.020752 \\
\hline 2 & 8 & -1.631952 & -0.762507 & -1.204750 \\
\hline 3 & 7 & -1.549165 & -0.100950 & 1.253576 \\
\hline 4 & 1 & -1.495791 & 0.113712 & 2.249781 \\
\hline 5 & 6 & -2.788747 & -0.843456 & -0.573582 \\
\hline 6 & 6 & -2.791821 & -0.489389 & 0.810253 \\
\hline 7 & 6 & -3.979270 & -0.564944 & 1.565049 \\
\hline 8 & 1 & -3.965517 & -0.302333 & 2.621340 \\
\hline 9 & 6 & -5.150369 & -0.975816 & 0.946606 \\
\hline 10 & 1 & -6.072242 & -1.035687 & 1.518010 \\
\hline 11 & 6 & -5.151178 & -1.319345 & -0.422948 \\
\hline 12 & 1 & -6.078512 & -1.640248 & -0.890326 \\
\hline 13 & 6 & -3.990219 & -1.257877 & -1.181647 \\
\hline 14 & 1 & -3.981661 & -1.527216 & -2.233812 \\
\hline 15 & 8 & 1.632580 & 0.067843 & 1.256204 \\
\hline 16 & 7 & 1.401501 & -1.036615 & -1.037405 \\
\hline 17 & 1 & 1.265833 & -1.585512 & -1.887065 \\
\hline 18 & 6 & 2.753487 & -0.427535 & 0.763962 \\
\hline 19 & 6 & 2.672237 & -1.091398 & -0.496892 \\
\hline 20 & 6 & 3.804115 & -1.705878 & -1.059800 \\
\hline 21 & 1 & 3.719634 & -2.221310 & -2.014820 \\
\hline 22 & 6 & 5.020110 & -1.634921 & -0.392026 \\
\hline 23 & 1 & 5.902821 & -2.099695 & -0.821659 \\
\hline 24 & 6 & 5.111223 & -0.960440 & 0.841855 \\
\hline 25 & 1 & 6.068333 & -0.915515 & 1.355336 \\
\hline 26 & 6 & 3.998718 & -0.360289 & 1.420361 \\
\hline 27 & 1 & 4.060876 & 0.144604 & 2.380292 \\
\hline 28 & 15 & 0.128120 & 2.069725 & -0.308415 \\
\hline 29 & 6 & -1.327215 & 2.848984 & -1.122145 \\
\hline 30 & 1 & -1.195161 & 3.933370 & -1.211604 \\
\hline 31 & 1 & -1.461021 & 2.410080 & -2.114730 \\
\hline
\end{tabular}




$\begin{array}{rrrrr}32 & 1 & -2.225439 & 2.639790 & -0.533623 \\ 33 & 6 & 0.362993 & 3.042372 & 1.239942 \\ 34 & 1 & 0.569687 & 4.096684 & 1.024283 \\ 35 & 1 & -0.542001 & 2.970194 & 1.850684 \\ 36 & 1 & 1.193876 & 2.601372 & 1.796243 \\ 37 & 6 & 1.561780 & 2.554977 & -1.356786 \\ 38 & 1 & 1.646899 & 3.646021 & -1.416074 \\ 39 & 1 & 2.478437 & 2.141265 & -0.926570 \\ 40 & 1 & 1.432361 & 2.141331 & -2.360383\end{array}$

D. $\quad c i s-\beta-(\operatorname{ap})_{2} \mathrm{Os}\left(\mathrm{P}[\mathrm{OMe}]_{3}\right)_{2}$

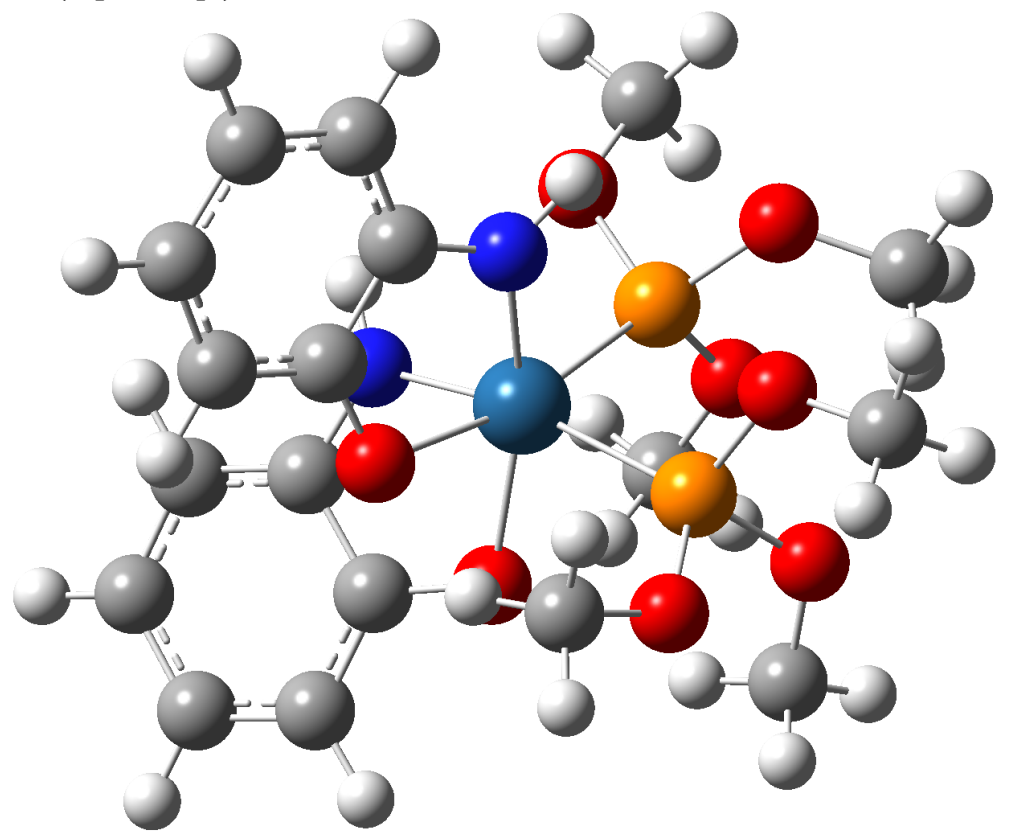

Energy of optimized structure $=-2187.64530890$ a.u.

$\mathrm{MOS}=-1.27(6),-1.35(7)$

Cartesian coordinates of optimized structure:

\begin{tabular}{|c|c|c|c|c|}
\hline \multirow{2}{*}{$\begin{array}{l}\text { Center } \\
\text { Number }\end{array}$} & \multirow{2}{*}{$\begin{array}{l}\text { Atomic } \\
\text { Number }\end{array}$} & \multicolumn{3}{|c|}{ Coordinates (Angstroms) } \\
\hline & & $\mathrm{x}$ & $\mathrm{Y}$ & $\mathrm{Z}$ \\
\hline 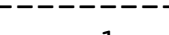 & & & & \\
\hline 1 & 76 & 0.084928 & 0.001348 & -0.082111 \\
\hline 2 & 8 & 1.826844 & -0.954928 & 0.662783 \\
\hline 3 & 7 & 0.272023 & -1.528444 & -1.340084 \\
\hline 4 & 1 & -0.444789 & -1.955836 & -1.921187 \\
\hline 5 & 8 & 0.408519 & 1.401071 & 1.447049 \\
\hline 6 & 7 & 1.416344 & 1.264804 & -0.949829 \\
\hline 7 & 1 & 1.464524 & 1.527325 & -1.930395 \\
\hline 8 & 15 & -1.592039 & 0.946622 & -1.297305 \\
\hline 9 & 15 & -1.436234 & -1.123117 & 1.315716 \\
\hline 10 & 6 & 2.327697 & -1.774912 & -0.235075 \\
\hline 11 & 6 & 1.498466 & -2.129016 & -1.357668 \\
\hline 12 & 6 & 1.942416 & -3.089665 & -2.293528 \\
\hline 13 & 1 & 1.300295 & -3.353372 & -3.131537 \\
\hline 14 & 6 & 3.193151 & -3.666092 & -2.145105 \\
\hline 15 & 1 & 3.548117 & -4.386698 & -2.876434 \\
\hline
\end{tabular}




$\begin{array}{rrrrr}16 & 6 & 4.012127 & -3.316375 & -1.049188 \\ 17 & 1 & 4.993421 & -3.773223 & -0.949552 \\ 18 & 6 & 3.584467 & -2.403345 & -0.095952 \\ 19 & 1 & 4.202452 & -2.141387 & 0.757864 \\ 20 & 6 & 1.571170 & 2.015899 & 1.271841 \\ 21 & 6 & 2.112054 & 2.030189 & -0.060491 \\ 22 & 6 & 3.227785 & 2.847866 & -0.346620 \\ 23 & 1 & 3.619460 & 2.876349 & -1.361078 \\ 24 & 6 & 3.838877 & 3.563461 & 0.671850 \\ 25 & 1 & 4.718314 & 4.163945 & 0.455470 \\ 26 & 6 & 3.336831 & 3.507737 & 1.988729 \\ 27 & 1 & 3.831329 & 4.070668 & 2.775978 \\ 28 & 6 & 2.210769 & 2.751759 & 2.287403 \\ 29 & 1 & 1.796299 & 2.722908 & 3.291241 \\ 30 & 8 & -0.981443 & 1.429216 & -2.736843 \\ 31 & 8 & -2.462462 & 2.241993 & -0.786786 \\ 32 & 8 & -2.829148 & 0.023189 & -1.825783 \\ 33 & 6 & -1.793370 & 3.338753 & -0.134267 \\ 34 & 1 & -2.577115 & 3.950838 & 0.317326 \\ 35 & 1 & -1.106654 & 2.975218 & 0.633940 \\ 36 & 1 & -1.243277 & 3.937522 & -0.868718 \\ 37 & 6 & -1.829108 & 1.966636 & -3.764472 \\ 38 & 1 & -1.165460 & 2.315928 & -4.557955 \\ 39 & 1 & -2.496817 & 1.192194 & -4.151851 \\ 40 & 1 & -2.421728 & 2.804538 & -3.383495 \\ 41 & 6 & -4.128574 & -0.013484 & -1.213731 \\ 42 & 1 & -4.669135 & -0.823020 & -1.708617 \\ 43 & 1 & -4.057508 & -0.217258 & -0.143376 \\ 44 & 1 & -4.649212 & 0.934585 & -1.374279 \\ 45 & 8 & -2.692885 & -0.264405 & 1.913783 \\ 46 & 8 & -2.259870 & -2.326943 & 0.589462 \\ 47 & 8 & -0.938202 & -1.762002 & 2.735598 \\ 48 & 6 & -3.242182 & -3.102342 & 1.299838 \\ 49 & 1 & -3.493574 & -3.944396 & 0.652241 \\ 50 & 1 & -2.837421 & -3.469585 & 2.247136 \\ 51 & 1 & -4.134333 & -2.500522 & 1.494514 \\ 52 & 6 & -2.467029 & 0.747547 & 2.916362 \\ 53 & 1 & -3.379346 & 1.346540 & 2.955014 \\ 54 & 1 & -2.292684 & 0.272812 & 3.886107 \\ 55 & 1 & -1.611770 & 1.375432 & 2.653294 \\ 56 & 6 & 0.271387 & -2.540121 & 2.851262 \\ 57 & 1 & 0.400190 & -2.733724 & 3.918235 \\ 58 & 0.161487 & -3.493045 & 2.320921 \\ 59 & 1.123471 & -1.988800 & 2.451616 \\ ------------------------------------------------\end{array}$




\section{E. Transition state for addition of $\mathrm{PMe}_{3}$ to $(\mathrm{ap})_{2} \mathrm{OsO}$}

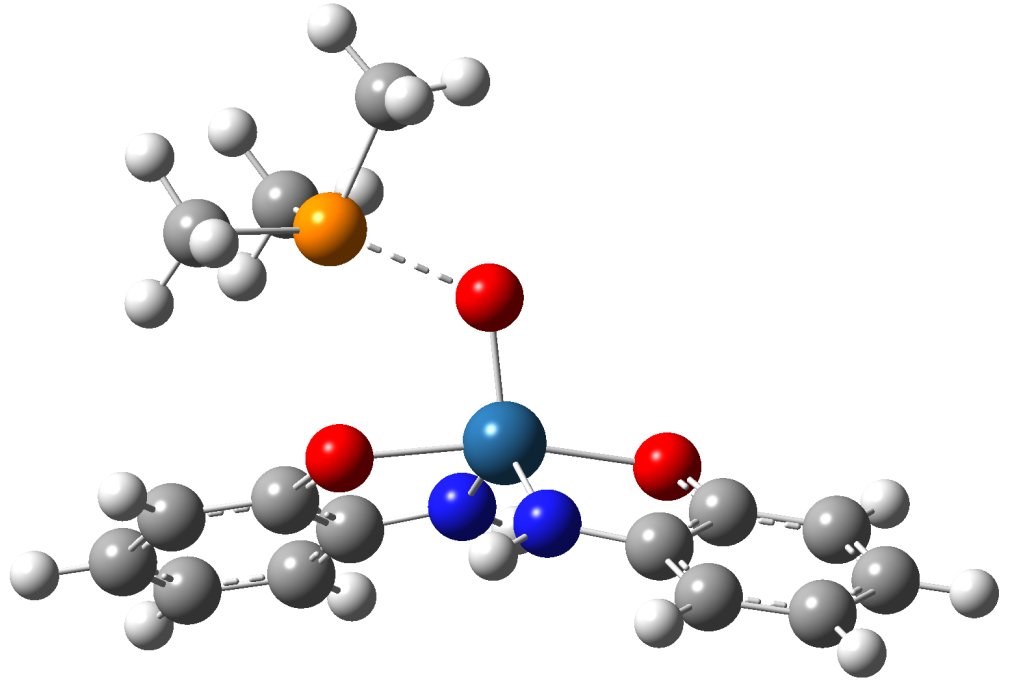

Energy of optimized structure $=-1350.33209280$ a.u.

MOS $=-1.80(6),-1.87(4)$

Imaginary frequency $=177.8 i \mathrm{~cm}^{-1}$

Cartesian coordinates of optimized structure:

\begin{tabular}{|c|c|c|c|c|}
\hline \multirow{2}{*}{$\begin{array}{l}\text { Center } \\
\text { Number }\end{array}$} & \multirow{2}{*}{$\begin{array}{l}\text { Atomic } \\
\text { Number }\end{array}$} & \multicolumn{3}{|c|}{ Coordinates (Angstroms) } \\
\hline & & $\mathrm{x}$ & $\mathrm{Y}$ & $\mathrm{z}$ \\
\hline 1 & 76 & 0.382657 & -0.046739 & -0.223783 \\
\hline 2 & 8 & 2.102653 & -0.355821 & -1.266096 \\
\hline 3 & 7 & 1.661341 & -0.020287 & 1.206515 \\
\hline 4 & 1 & 1.411238 & -0.054185 & 2.191894 \\
\hline 5 & 6 & 3.215509 & -0.244876 & -0.519945 \\
\hline 6 & 6 & 3.020595 & -0.069345 & 0.868124 \\
\hline 7 & 6 & 4.106386 & 0.022727 & 1.740378 \\
\hline 8 & 1 & 3.942183 & 0.154755 & 2.807777 \\
\hline 9 & 6 & 5.399720 & -0.056349 & 1.217906 \\
\hline 10 & 1 & 6.253592 & 0.016783 & 1.885363 \\
\hline 11 & 6 & 5.597472 & -0.230538 & -0.157823 \\
\hline 12 & 1 & 6.608220 & -0.289527 & -0.552662 \\
\hline 13 & 6 & 4.513377 & -0.331717 & -1.032855 \\
\hline 14 & 1 & 4.654721 & -0.472348 & -2.100330 \\
\hline 15 & 8 & -1.252086 & -0.175636 & 0.987918 \\
\hline 16 & 7 & -0.434237 & -1.622939 & -0.971317 \\
\hline 17 & 1 & -0.016636 & -2.199339 & -1.697684 \\
\hline 18 & 6 & -2.064613 & -1.213455 & 0.667392 \\
\hline 19 & 6 & -1.638337 & -2.043618 & -0.397107 \\
\hline 20 & 6 & -2.404707 & -3.146166 & -0.782242 \\
\hline 21 & 1 & -2.070197 & -3.781737 & -1.599016 \\
\hline 22 & 6 & -3.596157 & -3.420679 & -0.105268 \\
\hline 23 & 1 & -4.193187 & -4.279047 & -0.399561 \\
\hline 24 & 6 & -4.017043 & -2.603437 & 0.949165 \\
\hline 25 & 1 & -4.941254 & -2.829140 & 1.473721 \\
\hline 26 & 6 & -3.252531 & -1.500928 & 1.343925 \\
\hline 27 & 1 & -3.558626 & -0.870361 & 2.174077 \\
\hline 28 & 15 & -2.047696 & 2.326521 & 0.020719 \\
\hline 29 & 8 & -0.218757 & 1.537779 & -0.786819 \\
\hline
\end{tabular}




\begin{tabular}{|c|c|c|c|c|}
\hline 30 & 6 & -1.675800 & 4.026127 & -0.590644 \\
\hline 31 & 1 & -1.080968 & 4.562277 & 0.156042 \\
\hline 32 & 1 & -1.086301 & 3.955666 & -1.508408 \\
\hline 33 & 1 & -2.592002 & 4.595480 & -0.787111 \\
\hline 34 & 6 & -3.202254 & 1.637562 & -1.236089 \\
\hline 35 & 1 & -3.561188 & 0.658891 & -0.902182 \\
\hline 36 & 1 & -4.061723 & 2.298649 & -1.398413 \\
\hline 37 & 1 & -2.664927 & 1.506000 & -2.179371 \\
\hline 38 & 6 & -3.157501 & 2.666432 & 1.479433 \\
\hline 39 & 1 & -3.554556 & 1.718890 & 1.852082 \\
\hline 40 & 1 & -2.575325 & 3.133332 & 2.279857 \\
\hline 41 & 1 & -3.988433 & 3.330936 & 1.208111 \\
\hline
\end{tabular}

\section{F. (ap) $)_{2} \mathrm{Os}\left(\mathrm{OPMe}_{3}\right)$}

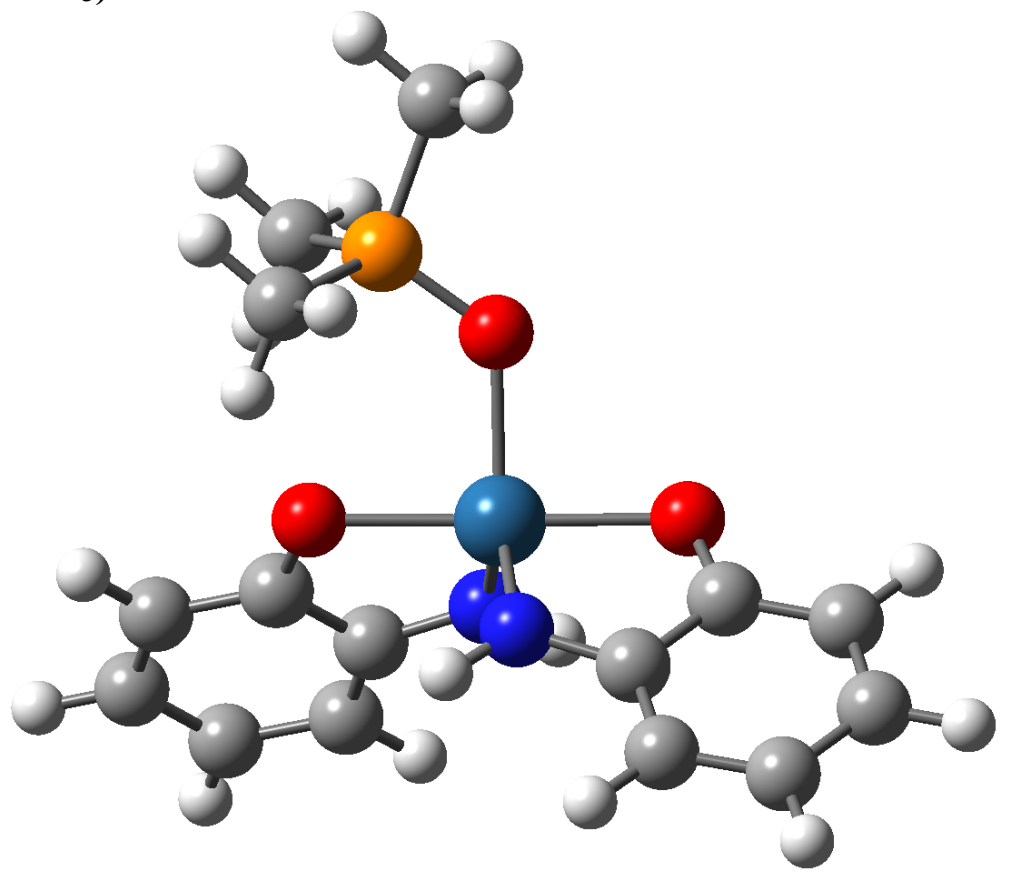

Energy of optimized structure $=-1350.38321862$ a.u.

MOS $=-1.63(10),-1.73(9)$

Cartesian coordinates of optimized structure:

\begin{tabular}{|c|c|c|c|c|}
\hline \multirow{2}{*}{$\begin{array}{l}\text { Center } \\
\text { Number }\end{array}$} & \multirow{2}{*}{$\begin{array}{l}\text { Atomic } \\
\text { Number }\end{array}$} & \multicolumn{3}{|c|}{ Coordinates (Angstroms) } \\
\hline & & $\mathrm{X}$ & $\mathrm{Y}$ & $\mathrm{Z}$ \\
\hline & & & & \\
\hline 1 & 76 & -1.096496 & 0.307313 & -0.205973 \\
\hline 2 & 8 & -1.492961 & -1.036434 & -1.756274 \\
\hline 3 & 7 & 0.314003 & 0.720549 & -1.395116 \\
\hline 4 & 1 & 1.032205 & 1.418963 & -1.202873 \\
\hline 5 & 6 & -0.546147 & -1.028825 & -2.684273 \\
\hline 6 & 6 & 0.502274 & -0.085209 & -2.525482 \\
\hline 7 & 6 & 1.554682 & 0.002779 & -3.441342 \\
\hline 8 & 1 & 2.348919 & 0.732390 & -3.291693 \\
\hline 9 & 6 & 1.564997 & -0.851997 & -4.542816 \\
\hline 10 & 1 & 2.375562 & -0.797586 & -5.263785 \\
\hline 11 & 6 & 0.526605 & -1.781632 & -4.717161 \\
\hline
\end{tabular}




\begin{tabular}{|c|c|c|c|c|}
\hline 12 & 1 & 0.542386 & -2.443829 & -5.579518 \\
\hline 13 & 6 & -0.523653 & -1.876473 & -3.806068 \\
\hline 14 & 1 & -1.327177 & -2.595276 & -3.938981 \\
\hline 15 & 8 & -0.722579 & 1.674179 & 1.383394 \\
\hline 16 & 7 & -0.487850 & -0.868075 & 1.147577 \\
\hline 17 & 1 & -0.406477 & -1.875885 & 1.016506 \\
\hline 18 & 6 & -0.114265 & 1.074447 & 2.409827 \\
\hline 19 & 6 & 0.061867 & -0.330643 & 2.320825 \\
\hline 20 & 6 & 0.687444 & -1.048884 & 3.342193 \\
\hline 21 & 1 & 0.815905 & -2.125681 & 3.249092 \\
\hline 22 & 6 & 1.139789 & -0.370302 & 4.475744 \\
\hline 23 & 1 & 1.630384 & -0.918693 & 5.274654 \\
\hline 24 & 6 & 0.961971 & 1.015569 & 4.577897 \\
\hline 25 & 1 & 1.319276 & 1.539500 & 5.460985 \\
\hline 26 & 6 & 0.338904 & 1.739867 & 3.559131 \\
\hline 27 & 1 & 0.209221 & 2.816703 & 3.632062 \\
\hline 28 & 15 & -3.597776 & 2.345372 & 0.312854 \\
\hline 29 & 8 & -3.041023 & 1.056834 & -0.359193 \\
\hline 30 & 6 & -5.214054 & 2.680968 & -0.453628 \\
\hline 31 & 1 & -5.077398 & 2.833386 & -1.528272 \\
\hline 32 & 1 & -5.869703 & 1.817668 & -0.306604 \\
\hline 33 & 1 & -5.680303 & 3.569991 & -0.016822 \\
\hline 34 & 6 & -3.872280 & 2.137376 & 2.097719 \\
\hline 35 & 1 & -2.901244 & 1.981880 & 2.573969 \\
\hline 36 & 1 & -4.359194 & 3.022149 & 2.521333 \\
\hline 37 & 1 & -4.504617 & 1.260214 & 2.266199 \\
\hline 38 & 6 & -2.547443 & 3.805989 & 0.068784 \\
\hline 39 & 1 & -1.588861 & 3.599880 & 0.554331 \\
\hline 40 & 1 & -2.392136 & 3.968459 & -1.002297 \\
\hline 41 & 1 & -3.014105 & 4.694411 & 0.507714 \\
\hline
\end{tabular}

